Article

\title{
Synthesis, Antiviral and Cytotoxic Activity of Novel Terpenyl Hybrid Molecules Prepared by Click Chemistry
}

\author{
Mariano Walter Pertino ${ }^{1,2, *}$, Erina Petrera ${ }^{3,4}$ (D), Laura Edith Alché ${ }^{3,4}$ and \\ Guillermo Schmeda-Hirschmann ${ }^{1,2}$ (D) \\ 1 Laboratorio de Química de Productos Naturales, Instituto de Química de Recursos Naturales, \\ Universidad de Talca, 3460000 Talca, Chile; schmeda@utalca.cl \\ 2 Programa de Investigación de Excelencia Interdisciplinaria en Química y Bio-orgánica de Recursos \\ Naturales (PIEI-QUIM-BIO), Universidad de Talca, 3460000 Talca, Chile \\ 3 Laboratorio de Virología, Departamento de Química Biológica, Facultad de Ciencias Exactas y Naturales, \\ Universidad de Buenos Aires, C1428BGA Buenos Aires, Argentina; epetrera@qb.fcen.uba.ar (E.P.); \\ lalche@qb.fcen.uba.ar (L.E.A.) \\ 4 Instituto de Química Biológica de la Facultad de Ciencias Exactas y Naturales (IQUIBICEN), \\ CONICET-Universidad de Buenos Aires, C1428EGA Buenos Aires, Argentina \\ * Correspondence: mwalter@utalca.cl; Tel.: +56-71-2418866
}

Received: 17 May 2018; Accepted: 1 June 2018; Published: 3 June 2018

\begin{abstract}
Naturally occurring terpenes were combined by click reactions to generate sixteen hybrid molecules. The diterpene imbricatolic acid (IA) containing an azide group was used as starting compound for the synthesis of all the derivatives. The alkyne group in the terpenes cyperenoic acid, dehydroabietinol, carnosic acid $\gamma$-lactone, ferruginol, oleanolic acid and aleuritolic acid was obtained by esterification using appropriate alcohols or acids. The hybrid compounds were prepared by combining the IA azide function with the different terpene-alkynes under click chemistry conditions. The cytotoxic activity of the terpene hybrids 1-16 was assessed against Vero cells and tumour cell lines (HEP-2, C6 and Raw 264.7). Compounds 1, 2, 3 and 7 showed cytotoxic activity against the tested cell lines. The antiviral activity of the compounds was evaluated against HSV-1 KOS, Field and B2006 strain. For the pairs of hybrid compounds formed between IA-diterpene (compounds 3-8, except for compound 7), a moderate activity was observed against the three HSV-1 strains with an interesting selectivity index ( $\left.\mathrm{SI} \geq 10, \mathrm{SI}=\mathrm{CC}_{50} / \mathrm{CE}_{50}\right)$ for some compounds.
\end{abstract}

Keywords: hybrid molecules; terpenes; antiviral; cytotoxicity; click chemistry

\section{Introduction}

Viruses are one of the main health problems worldwide due to the diseases they can cause and the low effectiveness of the current antiviral drugs [1]. One of the most common and highly infectious virus in humans is the herpes simplex virus type 1 (HSV-1). About 3500 million people worldwide have a prevalent infection of HSV-1 [2]. This virus is associated with a variety of diseases both mild and serious and even in some cases life-threatening [3]. Recent studies have demonstrated a direct relationship between HSV-1 infection with sporadic encephalitis [2] and Alzheimer's disease [4]. Although there are drugs approved for the treatment of HSV, such as acyclovir, routine use of this drug generates resistance, especially in immunocompromised patients and patients with stem cell transplants [5], therefore, there is a continuing need to find new chemical entities to be used as antiviral agents. 
Terpenes are secondary metabolites that occur across a wide range of plant tissue types and present several biological activities. Some of them have shown remarkable antiviral [6-8] and cytotoxic effects [9-11]. In previous works, we prepared series of semisynthetic derivatives of a large number of terpenes in the search for biologically active derivatives [12-15]. The semisynthetic strategies that we have used in the search for bioactive compounds have been varied. Among them, we described the synthesis of imbricatolic acid dimers [16] and hybrid terpene and quinone molecules [17].

Molecular hybridization is a strategy for the discovery of new drugs that consists in the fusion of two bioactive compounds to generate a new entity called a hybrid molecule [18]. In the last two decades molecular hybridization has gained importance as a valid tool in the search for bioactive compounds. A recently review highlights hybrid molecules as privileged scaffolds to identify potential bioactive agents [19]. In that review almost 200 hybrid molecules of different nature, with high structural variety and significant activities were presented. Adopting this approach, Belluti et al. [20] synthesized ten stilbene-coumarin hybrids and evaluated their antiproliferative activity against lung carcinoma H460, squamous cell carcinoma A431 and melanoma JR8 tumour cells. Some of the prepared hybrids showed better antiproliferative activity than the reference compound (resveratrol) with pro-apoptotic activities on $\mathrm{H} 460$ cells. Other compounds that showed potential anticancer activity were hybrids formed by two phenolic compounds. Sashidhara et al. [21] prepared a series of 21 coumarin-chalcone hybrids with significant antiproliferative activity for several of the compounds evaluated. Recently, a synthetic strategy has been reported to combine flavonoid dimers and trimers linked by triazole ring using click chemistry [22].

In this report, we used triazole rings as linkers due to the synthetic advantages of this reaction. The triazole ring also has high aromatic stabilization that make it stable against adverse conditions including hydrolysis. In addition, in the formation of hybrid molecules triazoles can act as a simple linker or spacer or, in some cases, as an active biological entity [23]. There are numerous reports where the click chemistry technique has been used to prepare hybrid molecules with various biological effects [24-26].

Terpene hybrids reported in bibliography include hybrids between terpene-benzoylphenyl urea (BPU) [27], hybrid terpene-amino acid [28,29], hybrid terpene-quinone [17,30], hybrid terpene-anti-inflammatory agents [31], hybrid terpene-NSAID [32], among others. However, to date there is little information on the possible biological effect that hybrid molecules formed by the fusion of two different terpenes may have. In this work, our aim was to synthesize hybrid molecules by click chemistry reactions between the diterpene imbricatolic acid and several terpenes, including cyperenoic acid, dehydroabietinol, carnosic acid $\gamma$-lactone, ferruginol, aleuritolic acid and oleanolic acid.

\section{Results and Discussion}

Naturally occurring terpenes were combined by click chemistry to obtain sixteen hybrid molecules. The diterpene imbricatolic acid (IA) with an azide group was used as starting compound for the synthesis of all the derivatives. The azide group was installed at the C-15 position of imbricatolic acid according to our previous work [16]. Briefly, IA was methylated, tosylated, and finally treated with $\mathrm{NaN}_{3}$ to form the azide derivative (Scheme 1).

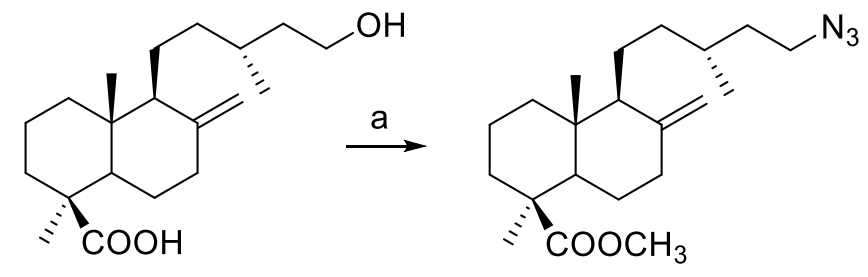

Scheme 1. Preparation of IA-azide derivative. Reagents and conditions: (a) (i) $\mathrm{CH}_{2} \mathrm{~N}_{2}, \mathrm{Et}_{2} \mathrm{O}, 93 \%$; (ii) $\mathrm{TsCl}$, pyridine, $76 \%$; (iii) $\mathrm{NaN}_{3}, \mathrm{DMF}, 88 \%$. 
The alkyne groups in the terpenes cyperenoic acid, dehydroabietinol, carnosic acid $\gamma$-lactone, ferruginol, oleanolic acid and aleuritolic acid were obtained by esterification using appropriate alcohols or acids according our previous work $[13,33,34]$. The alcohols used were propargyl alcohol and 3-butyn-1-ol. The acids used were 4-pentynoic acid and 5-hexynoic acid. Compounds 1-16 were prepared by combining the IA-azide with the different terpene-alkynes by click chemistry (Scheme 2).
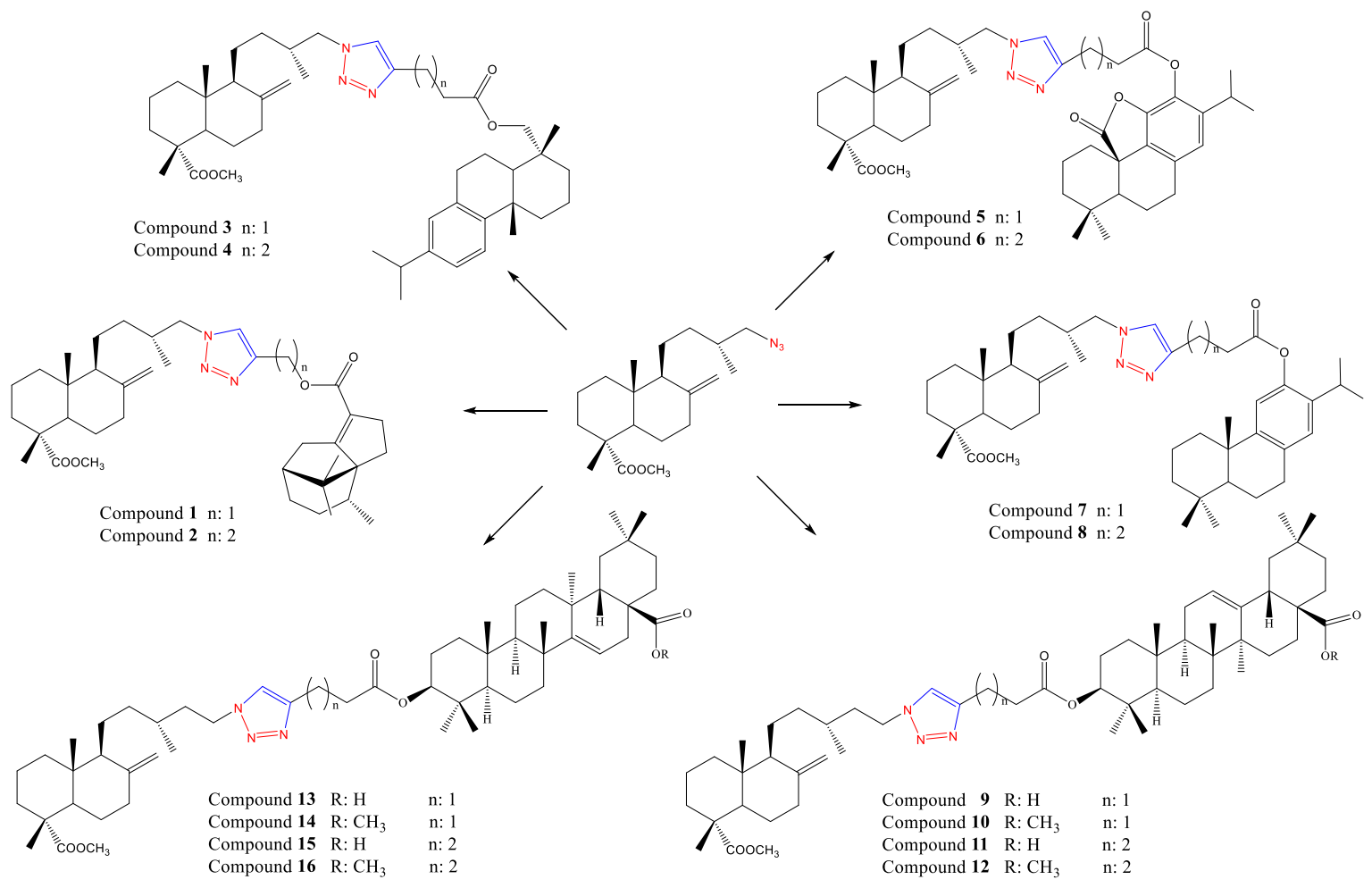

Scheme 2. Preparation of derivatives 1-16. Reagents and conditions: appropriate alkyne-terpene, $\mathrm{CuSO}_{4} \cdot 5 \mathrm{H}_{2} \mathrm{O}$, sodium ascorbate, $\mathrm{t}-\mathrm{BuOH} / \mathrm{H}_{2} \mathrm{O} 1: 1,53-83 \%$ yield.

\subsection{Cytotoxicity Assay}

The cytotoxic activity of the hybrid terpenes 1-16 against Vero and selected tumour cell lines (HEP-2, C6 and Raw 264.7) was determined and the 50\% cytotoxic concentration $\left(\mathrm{CC}_{50}\right)$ of each compound was calculated. Only compounds 1, 2, 3 and 7 showed cytotoxic activity against the tested cell lines. All other compounds should be regarded as non-cytotoxic, with $\mathrm{CC}_{50}$ values $>1000 \mu \mathrm{M}$ on all evaluated cell lines. The most relevant results are summarized in Table 1.

Table 1. Cytotoxic activity of compounds 1-4 and 7-8 against Vero and selected tumor cell lines ${ }^{a}$.

\begin{tabular}{ccccc}
\hline \multirow{2}{*}{ Compound } & \multicolumn{4}{c}{$\mathrm{CC}_{\mathbf{5 0}}(\boldsymbol{\mu M})$} \\
\cline { 2 - 5 } & Vero & HEP-2 & C6 & Raw 264.7 \\
\hline $\mathbf{1}$ & $623.9 \pm 5$ & $229.7 \pm 1.8$ & $>1000$ & $>1000$ \\
$\mathbf{2}$ & $216.2 \pm 2$ & $48.1 \pm 1.1$ & $354.9 \pm 2.5$ & $300 \pm 1.9$ \\
$\mathbf{3}$ & $667.5 \pm 4.2$ & $411.8 \pm 2.1$ & $>1000$ & $>1000$ \\
$\mathbf{4}$ & $>1000$ & $>1000$ & $>1000$ & $>1000$ \\
$\mathbf{7}$ & $680 \pm 4.8$ & $700 \pm 5.8$ & $552.5 \pm 4$ & $552.5 \pm 3.9$ \\
$\mathbf{8}$ & $>1000$ & $>1000$ & $>1000$ & $>1000$ \\
\hline \multicolumn{5}{c}{}
\end{tabular}

${ }^{a}$ For the cell lines used, see the text. 
The pairs of compounds 1-2, 3-4 and 7-8 differ in one $\mathrm{CH}_{2}$ unit in the linker. The hybrid between imbricatolic acid and cyperenoic acid (compounds $\mathbf{1}$ and 2) showed selective cytotoxicity. Both compounds were more cytotoxic against HEP-2 cells with CC50 values of 229.7 and $48.1 \mu \mathrm{M}$, respectively. Compound 2 was the most cytotoxic compound among the series of derivatives against all cell lines used. Compound $\mathbf{1}$ did not show cytotoxicity against C6 and Raw 264.7 cells (see Table 1 ). For the pairs of compounds 3-4 (hybrid between IA-dehydroabietinol) and 7-8 (hybrid between IA-ferruginol), the activity decreased with linker length. Compound 3 presented some effect against Vero and HEP-2 cells, but not against C6 and Raw 264.7 cells. Compound 7 showed weak activity on all cell lines, with $\mathrm{CC}_{50}$ values ranging from 552.5 to $700 \mu \mathrm{M}$. Compounds 4 and 8 were not cytotoxic.

\subsection{Antiviral Activity Screening}

Most of the compounds presented moderate cytotoxicity or were inactive, which renders them suitable as potential antivirals. The antiviral activity of the compounds 1-16 was assessed against three different strains of HSV-1 (KOS, Field and B2006 strain). First, the 50\% cytotoxic concentration $\left(\mathrm{CC}_{50}\right)$ of the compounds was calculated using Vero cells. It is prerequisite that antiviral compounds do not cause significant damage to host cells. The results are presented in Table 2. The most cytotoxic hybrid was compound $2\left(\mathrm{CC}_{50}: 216.2 \mu \mathrm{M}\right)$, followed by compound 3 with $\mathrm{CC}_{50}: 667.5 \mu \mathrm{M}$. All other hybrids were not cytotoxic.

Table 2. Antiviral activity of hit compounds against wild-type and ACV-resistant HSV-1 strains. $\mathrm{EC}_{50} \mathrm{~S}$ were calculated by nonlinear regression.

\begin{tabular}{cccccccc}
\hline \multirow{2}{*}{ Compound } & \multirow{2}{*}{$\mathbf{C C}_{\mathbf{5 0}}{ }^{a}(\boldsymbol{\mu M})$} & \multicolumn{3}{c}{ EC $_{\mathbf{5 0}}(\boldsymbol{\mu M})$} \\
\cline { 2 - 8 } & & KOS & Field & B2006 & KOS & Field & B2006 \\
\hline $\mathbf{2}$ & $216.2 \pm 2$ & $118.4 \pm 2.3$ & $157.9 \pm 1.9$ & $226.3 \pm 2.1$ & 1.8 & 1.3 & 0.9 \\
$\mathbf{3}$ & $667.5 \pm 4.2$ & $107.9 \pm 1.2$ & $140.2 \pm 1.2$ & $122.1 \pm 1.2$ & 6.2 & 4.76 & 5.5 \\
$\mathbf{4}$ & $>1000$ & $109 \pm 1.0$ & $137.5 \pm 1.9$ & $110 \pm 1.0$ & $>9.2$ & $>7.2$ & $>9$ \\
$\mathbf{5}$ & $>1000$ & $105.1 \pm 1.1$ & $140.3 \pm 2.1$ & $112.1 \pm 1.1$ & $>9.5$ & $>7.1$ & $>8.9$ \\
$\mathbf{6}$ & $>1000$ & $96.2 \pm 1.2$ & $137.8 \pm 1.4$ & $120 \pm 1.5$ & $>10.4$ & $>7.3$ & $>8.3$ \\
$\mathbf{8}$ & $>1000$ & $99.3 \pm 1.5$ & $132.4 \pm 1.1$ & $105.9 \pm 1.9$ & $>10$ & $>7.6$ & $>9.4$ \\
$\mathrm{ACV}^{b}$ & $>1000$ & $8.2 \pm 1.0$ & $73 \pm 1.0$ & $50 \pm 2.0$ & $>121.9$ & $>13.7$ & $>20$ \\
\hline
\end{tabular}

${ }^{a}$ For Vero cells. ${ }^{b} \mathrm{ACV}$ : Acyclovir, reference compound.

The results showed similar antiviral activity against the HSV-1 KOS strain, with $\mathrm{EC}_{50}$ values of the hit compounds between $96.2-118.4 \mu \mathrm{M}$ and with selectivity indexes $\left(\mathrm{SI}=\mathrm{CC}_{50} / \mathrm{CE}_{50}\right)>9$ for compounds 4 and 5, and $>10$ for compounds 6 and 8, respectively. For the HSV-1 field strain, the $\mathrm{EC}_{50}$ values for the hit compounds were in the range 132.4-157.9 $\mu \mathrm{M}$, with compounds 4, 5, 6 and 8 showing the best selectivity index ( $>7$ ). For HSV-1 B2006, only compound 2 showed a significant difference in activity compared to the other hybrids with an $\mathrm{EC}_{50}$ value about half that of the other products $\left(\mathrm{EC}_{50}=226.3\right.$ for compound 2 vs. $\mathrm{EC}_{50}$ between 105.9-122.1 for the other hybrids).

Comparison of the results on the different viruses showed interesting information. For the pairs of compounds 1-2 and 7-8 (differing in one $\mathrm{CH}_{2}$ unit in the linker), activity was found for compounds 2 and 8, suggesting that the length of the linker influences the antiviral effect of this group of compounds. However, no difference were observed against any of the three HSV-1 strains for the pairs IA-diterpene (compounds 3-8, except for compound 7). The hybrids IA-triterpene (compounds 9-16) were inactive.

\section{Experimental}

\subsection{General Procedures}

Optical rotations were measured in $\mathrm{CHCl}_{3}$ at $20^{\circ} \mathrm{C}$ on a Jasco DIP 370 polarimeter (Jasco Analytical Instruments, Easton, MD, USA). IR spectra were recorded on a Nicolet Nexus 470 FT-IR instrument 
(Thermo Electron Corporation, Whaltham, MA, USA). The NMR spectra were recorded on a Bruker Avance 400 spectrometer (Bruker, Rheinstetten, Germany) at $400 \mathrm{MHz}$ for ${ }^{1} \mathrm{H}$ and $100 \mathrm{MHz}$ for ${ }^{13} \mathrm{C}$ in $\mathrm{CDCl}_{3}$. Chemical shifts are given in ppm with TMS as the internal standard. High-resolution mass spectra were measured on a VG Micromass ZAB-2F at $70 \mathrm{eV}$ (Varian Inc., Palo Alto, CA, USA). Merck silica gel (0.063-0.2) was used for column chromatography, pre-coated Si gel plates (Merck, Kieselgel $60 \mathrm{~F}_{254}, 0.25 \mathrm{~mm}$ ) were used for TLC analysis. TLC spots were visualized by spraying the chromatograms with $p$-anisaldehyde-ethanol-acetic acid- $\mathrm{H}_{2} \mathrm{SO}_{4}(2: 170: 20: 10 \mathrm{v} / \mathrm{v})$ and heating at $110{ }^{\circ} \mathrm{C}$ for $3 \mathrm{~min}$. Reagents: N,N-Dicyclohexylcarbodiimide (DCC) and dimethylaminopyridine (DMAP) were from Merck (Schuchardt, Germany). Propargyl alcohol, 3-butyn-1-ol, 4-pentynoic acid and 5-hexynoic acid were from Aldrich (Steinheim, Germany). Copper (II) sulphate pentahydrate was from Aldrich (St. Louis, MO, USA) and sodium ascorbate was from Sigma (St. Louis, MO, USA).

\subsection{Preparation of Derivatives}

\subsubsection{Starting Compounds}

Imbricatolic acid was isolated from the resin of Araucaria araucana [28]. Cyperenoic acid and acetyl aleuritolic acid were obtained from Jatropha isabelli [11]. Dehydroabietic acid was prepared from commercial rosin as previously described [33]. Carnosic acid was isolated from the aerial parts of Rosmarinus officinalis [35]. Ferruginol was isolated from the stem bark of Prumnopitys andina [36] and oleanolic acid from the aerial parts of Fabiana imbricata [37].

\subsubsection{Preparation of Terpene-Alkynyl Esters}

Esterification of terpenes was performed using DCC/DMAP and an appropriate alcohol (propargyl alcohol or 3-butyn-1-ol) for cyperenoic acid, or acid (4-pentynoic or 5-hexynoic acid) for dehydroabietinol (obtained by reduction of dehydroabietic acid), carnosic acid, ferruginol, oleanolic acid and aleuritolic acid (obtained by deacetylation of acetyl aleuritolic acid) according to references $[13,33,34]$. In previous work we reported the formation of carnosic acid lactone in the esterification reaction leading to carnosic acid $\gamma$-lactone [35]. Briefly, cyperenoic acid or alkynyl acid (1 equiv.) was dissolved in dry $\mathrm{CH}_{2} \mathrm{Cl}_{2}$ at room temperature under constant stirring. Then, DCC (1 equiv.) was added, followed by a catalytic amount of DMAP and alkynyl alcohol or terpene alcohol (1 equiv.) dissolved in dry $\mathrm{CH}_{2} \mathrm{Cl}_{2}$. The reaction was stopped by adding $\mathrm{H}_{2} \mathrm{O}$, extracted with $\mathrm{CH}_{2} \mathrm{Cl}_{2}$, dried over $\mathrm{Na}_{2} \mathrm{SO}_{4}$, concentrated and purified by column chromatography on silica gel (60-81\% yield).

\subsubsection{Synthesis of Hybrid Derivatives 1-16}

The terpene-alkynyl esters ( 1 equiv.) and the IA-azide (1 equiv.) were dissolved in $t$ - $\mathrm{BuOH} / \mathrm{H}_{2} \mathrm{O}$ (1:1), followed by the addition of $\mathrm{CuSO}_{4} \cdot 5 \mathrm{H}_{2} \mathrm{O}(2 \mathrm{~mol} \%)$ and sodium ascorbate $(10 \mathrm{~mol} \%)$. The mixture was stirred at room temperature for $24 \mathrm{~h}$. The reaction was stopped by adding $\mathrm{H}_{2} \mathrm{O}$, extracted with $\mathrm{CH}_{2} \mathrm{Cl}_{2}$, dried over anhydrous $\mathrm{Na}_{2} \mathrm{SO}_{4}$, concentrated and purified by column chromatography on silica gel (53-83\% yield). The compounds 10, 12, 14 and 16 were obtained by methylation using diazomethane in diethyl ether $\left(\mathrm{Et}_{2} \mathrm{O}\right)$ of the compounds $9,11,13$ and 15 , respectively ( $88 \%-94 \%$ yield).

Compound (1). Colorless resin; $[\alpha]_{D}^{20}+21\left(c 0.059, \mathrm{CHCl}_{3}\right)$; IR (film) $v_{\max } 2949,2864,1720,1707$, 1660, 1456, 1255, $753 \mathrm{~cm}^{-1} ;{ }^{1} \mathrm{H}-\mathrm{NMR}\left(400 \mathrm{MHz}, \mathrm{CDCl}_{3}\right) \delta 7.59\left(\mathrm{~s}, 1 \mathrm{H}, \mathrm{H}-1^{\prime}\right), 5.24\left(\mathrm{~s}, 2 \mathrm{H}, \mathrm{H}-3^{\prime}\right), 4.81$ (s, 1H, H-17), 4.41 (s, 1H, H-17), 4.37-4.27 (m, 2H, H-15), 3.59 (s, 3H, OMe), 280-2.70 (m, 1H, H-3"), 2.69-2.57 (m, 2H, H-3" and H-6" $), 2.39-2.36(\mathrm{~m}, 1 \mathrm{H}, \mathrm{H}-7), 2.20-2.10\left(\mathrm{~m}, 2 \mathrm{H}, \mathrm{H}-3\right.$ and H-6" $\left.{ }^{\prime \prime}\right), 1.16(\mathrm{~s}, 3 \mathrm{H}$, H-18), 0.95 (s, 3H, H-13") $, 0.93(\mathrm{~d}, J=6.6 \mathrm{~Hz}, 3 \mathrm{H}, \mathrm{H}-16), 0.80\left(\mathrm{~d}, J=6.5 \mathrm{~Hz}, 3 \mathrm{H}, \mathrm{H}-14^{\prime \prime}\right), 0.76(\mathrm{~s}, 3 \mathrm{H}$, $\left.\mathrm{H}-12^{\prime \prime}\right), 0.46$ (s, 3H, H-20). ${ }^{13} \mathrm{C}-\mathrm{NMR}\left(\mathrm{CDCl}_{3}\right): \delta 177.83,170.67,165.42,148.33,143.50,123.73,123.06$, $106.38,68.06,57.27,56.63,56.42,51.23,48.67,48.22,44.38,41.74,40.45,39.27,38.85,38.33,37.18,36.69$, $35.97,35.88,31.30,31.15,28.92,27.93,27.02,26.33,25.80,21.09,20.05,19.56,19.36,18.05,12.65$. HREIMS $m / z 620.4486[\mathrm{M}+\mathrm{H}]^{+}\left(\right.$calcd for $\left.\mathrm{C}_{38} \mathrm{H}_{58} \mathrm{~N}_{3} \mathrm{O}_{4}, 620.4437\right)$. 
Compound (2). Colorless resin; $[\alpha]_{D}^{20}+18\left(c\right.$ 0.032, $\left.\mathrm{CHCl}_{3}\right)$; IR (film) $v_{\max } 2927,2867,1722,1704$, 1663, 1462, 1258, $759 \mathrm{~cm}^{-1} ;{ }^{1} \mathrm{H}-\mathrm{NMR}\left(400 \mathrm{MHz}, \mathrm{CDCl}_{3}\right) \delta 7.32$ (s, 1H, H-1'), $4.82(\mathrm{~s}, 1 \mathrm{H}, \mathrm{H}-17), 4.42$ (s, 1H, H-17), 4.41-4.28 (m, 4H, H-15 and H-4'), 3.60 (s, 3H, OMe), $3.10\left(\mathrm{t}, J=6.7 \mathrm{~Hz}, 2 \mathrm{H}, \mathrm{H}-3^{\prime}\right), 2.82-2.70$ $\left(\mathrm{m}, 1 \mathrm{H}, \mathrm{H}-3^{\prime \prime}\right), 2.68-2.54\left(\mathrm{~m}, 2 \mathrm{H}, \mathrm{H}-3^{\prime \prime}\right.$ and $\left.\mathrm{H}-6^{\prime \prime}\right), 2.39-2.36(\mathrm{~m}, 1 \mathrm{H} \mathrm{H}-7), 2.17-2.13(\mathrm{~m}, 2 \mathrm{H}, \mathrm{H}-3$ and H-6") 1.16 (s, 3H, H-18), 0.96 (s, 3H, H-13" ) 0.93 (d, J = 6.5 Hz, 3H H-16), 0.82 (d, J = 6.5 Hz, 3H, $\left.\mathrm{H}-14^{\prime \prime}\right), 0.78$ (s, 3H, H-12") 0.47 (s, 3H, H-20). ${ }^{13} \mathrm{C}-\mathrm{NMR}\left(101 \mathrm{MHz}, \mathrm{CDCl}_{3}\right) \delta 177.86,169.84,165.44$, 148.36, 144.51, 123.37, 121.27, 106.39,67.99, 62.70, 56.69, 56.45, 51.26, 48.57, 48.25, 44.41, 41.75, 40.48, 39.30, 38.88, 38.36, 37.38, 36.76, 35.96, 35.94, 31.28, 31.23, 28.94, 28.02, 27.08, 26.35, 25.85, 25.80, 21.11, $20.07,19.59,19.37,18.11,12.69$. HREIMS $m / z 656.4493[\mathrm{M}+\mathrm{Na}]^{+}$(calcd for $\mathrm{C}_{39} \mathrm{H}_{59} \mathrm{~N}_{3} \mathrm{NaO}_{4}, 656.4403$ ).

Compound (3). Colorless resin; $[\alpha]_{D}^{20}+28\left(c 0.050, \mathrm{CHCl}_{3}\right)$; IR (film) $v_{\max } 2927,2867,1732,1710$, 1661, 1462, 1152, $756 \mathrm{~cm}^{-1} ;{ }^{1} \mathrm{H}-\mathrm{NMR}\left(400 \mathrm{MHz}, \mathrm{CDCl}_{3}\right) \delta 7.28\left(\mathrm{~s}, 1 \mathrm{H}, \mathrm{H}-1^{\prime}\right), 7.16(\mathrm{~d}, J=8.3 \mathrm{~Hz}, 1 \mathrm{H}$, H-11"), 6.98 (brd, J = 8.2 Hz, 1H, H-12"'), 6.88 (s, 1H, H-14"), 4.82 (s, 1H, H-17), 4.44 (s, 1H, H-17), 4.32-4.22 (m, 2H, H-15), 3.96 (d, J = 10.9 Hz, 1H, H-18' ), 3.73 (d, J = $\left.10.9 \mathrm{~Hz}, 1 \mathrm{H}, \mathrm{H}-18^{\prime \prime}\right), 3.60$ (s, 3H, OMe), $3.01\left(\mathrm{t}, J=7.3 \mathrm{~Hz}, 2 \mathrm{H}, \mathrm{H}-4^{\prime}\right), 2.93-2.62\left(\mathrm{~m}, 3 \mathrm{H}, \mathrm{H}-7^{\prime \prime}\right.$ and $\left.\mathrm{H}-15^{\prime \prime}\right), 2.71\left(\mathrm{t}, J=7.4 \mathrm{~Hz}, 2 \mathrm{H}, \mathrm{H}-3^{\prime}\right)$, 2.40-2.37 (m, 1H, H-7), 2.32-2.23 (m, 1H, H-1 $\left.\beta^{\prime \prime}\right), 2.18-2.15$ (m, 1H, H-3), 1.22 (d, J = 6.9 Hz, 6H, H-16" and H-17"), $1.20\left(\mathrm{~s}, 3 \mathrm{H}, \mathrm{H}-20^{\prime \prime}\right), 1.17$ (s, 3H, H-18), 0.93 (d, $\left.J=6.5 \mathrm{~Hz}, 3 \mathrm{H}, \mathrm{H}-16\right), 0.92\left(\mathrm{~s}, 3 \mathrm{H}, \mathrm{H}-19^{\prime \prime}\right), 0.48$ (s, 3H, H-20). ${ }^{13} \mathrm{C}-\mathrm{NMR}\left(101 \mathrm{MHz}, \mathrm{CDCl}_{3}\right) \delta 177.83,172.98,148.33,147.14,146.34,145.67,134.71,126.95$, $124.36,123.98,121.00,106.39,72.76,56.64,56.43,51.21,48.47,44.50,44.39,40.45,39.27,38.85,38.34,37.49$, $37.25,36.88,35.91,35.64,33.80,33.51,31.13,30.32,28.91,26.34,25.44,24.06(2 \mathrm{C}), 21.14,21.08,20.05$, 19.56, 19.10, 18.60, 17.50, 12.66. HREIMS $m / z$ 714.5302 [M + H] ${ }^{+}$(calcd for $\mathrm{C}_{45} \mathrm{H}_{68} \mathrm{~N}_{3} \mathrm{O}_{4}, 714.5210$ ).

Compound (4). Colorless resin; $[\alpha]_{D}^{20}+26\left(c 0.191, \mathrm{CHCl}_{3}\right)$; IR (film) $v_{\max } 2930,2867,1722,1718$, $1678,1462,1155,753 \mathrm{~cm}^{-1} ;{ }^{1} \mathrm{H}-\mathrm{NMR}\left(400 \mathrm{MHz}, \mathrm{CDCl}_{3}\right) \delta 7.17\left(\mathrm{~d}, J=8.1 \mathrm{~Hz}, 1 \mathrm{H}, \mathrm{H}-11^{\prime \prime}\right), 7.14(\mathrm{~s}, 1 \mathrm{H}$, H-1'), 6.97 (brd, $\left.J=8.1 \mathrm{~Hz}, 1 \mathrm{H}, \mathrm{H}-12^{\prime \prime}\right), 6.86\left(\mathrm{~s}, 1 \mathrm{H}, \mathrm{H}-14^{\prime \prime}\right), 4.80$ (s, 1H, H-17), 4.42 (s, 1H, H-17),

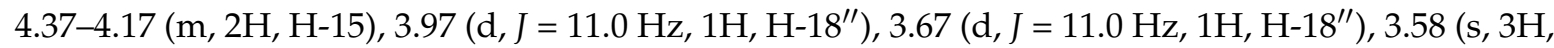
OMe), 2.94-2.58 (m, 5H, H-7", $\mathrm{H}-15^{\prime \prime}$ and $\left.\mathrm{H}-5^{\prime}\right), 2.42-2.21\left(\mathrm{~m}, 4 \mathrm{H}, \mathrm{H}-7, \mathrm{H}-1 \beta^{\prime \prime}\right.$ and $\left.\mathrm{H}-3^{\prime}\right), 2.19-2.15$ (m, 1H, H-3), 1.19 (d, J = 6.5 Hz, 6H, H-16" and H-17"), 1.18 (s, 3H, H-20"), 1.15 (s, 3H, H-18), 0.92 (d, $J=6.5 \mathrm{~Hz}, 3 \mathrm{H}, \mathrm{H}-16), 0.91$ (s, 3H, H-19") 0.46 (s, 3H, H-20). ${ }^{13} \mathrm{C}-\mathrm{NMR}\left(101 \mathrm{MHz}, \mathrm{CDCl}_{3}\right) \delta 177.73$, 173.41, 148.26, 147.14, 146.97, 145.61, 134.71, 126.90, 124.28, 123.91, 120.68, 106.32, 72.37, 56.58, 56.36, $51.13,48.38,44.31,40.38,39.20,38.79,38.34,38.27,37.44,37.21,36.83,35.85,35.59,33.59,33.44,31.09$, $30.25,28.85,26.28,25.39,24.97,24.68,23.99$ (2C), 21.03, 19.99, 19.52, 19.01, 18.55, 17.52, 12.59. HREIMS $m / z 728.5318[\mathrm{M}+\mathrm{H}]^{+}$(calcd for $\mathrm{C}_{46} \mathrm{H}_{70} \mathrm{~N}_{3} \mathrm{O}_{4}, 728.5366$ ).

Compound (5). Colorless resin; $[\alpha]_{D}^{20}+27\left(c 0.053, \mathrm{CHCl}_{3}\right)$; IR (film) $v_{\max } 2927,2870,1754,1718$, 1641, 1462, 1136, $756 \mathrm{~cm}^{-1}$; ${ }^{1} \mathrm{H}-\mathrm{NMR}\left(400 \mathrm{MHz}, \mathrm{CDCl}_{3}\right) \delta 7.37\left(\mathrm{~s}, 1 \mathrm{H}, \mathrm{H}-1^{\prime}\right), 6.91\left(\mathrm{~s}, 1 \mathrm{H}, \mathrm{H}-14^{\prime \prime}\right), 6.78$ (s, 1H, H-11") 4.82 (s, 1H, H-17), 4.43 (s, 1H, H-17), 4.34-4.26 (m, 2H, H-15), 3.61 (s, 3H, OMe), 3.15 $\left(\mathrm{t}, J=7.2 \mathrm{~Hz}, 2 \mathrm{H}, \mathrm{H}-4^{\prime}\right), 3.00\left(\mathrm{t}, J=7.2 \mathrm{~Hz}, 2 \mathrm{H}, \mathrm{H}-3^{\prime}\right), 2.93-2.87\left(\mathrm{~m}, 1 \mathrm{H}, \mathrm{H}-15^{\prime \prime}\right), 2.84-2.73\left(\mathrm{~m}, 2 \mathrm{H}, \mathrm{H}-7^{\prime \prime}\right)$, 2.44-2.35 (m, 1H, H-7), 2.21-2.08 (m, 2H, H-3 and H-1 $\left.\beta^{\prime \prime}\right), 1.17$ (s, 3H, H-18), 1.16 (s, 3H, H-20" $), 1.12$ $\left(\mathrm{d}, J=6.9 \mathrm{~Hz}, 3 \mathrm{H}, \mathrm{H}-16^{\prime \prime}\right), 1.10\left(\mathrm{~d}, J=6.9 \mathrm{~Hz}, 3 \mathrm{H}, \mathrm{H}-17^{\prime \prime}\right), 0.94(\mathrm{~d}, J=6.6 \mathrm{~Hz}, 3 \mathrm{H}, \mathrm{H}-16), 0.93(\mathrm{~s}, 3 \mathrm{H}$, $\left.\mathrm{H}-18^{\prime \prime}\right), 0.91$ (s, 3H, H-19") 0.48 (s, 3H, H-20). ${ }^{13} \mathrm{C}-\mathrm{NMR}\left(101 \mathrm{MHz}, \mathrm{CDCl}_{3}\right) \delta 177.90,171.96,148.88$, 148.36, 146.21, 146.09, 136.62, 133.24, 126.97, 121.40, 117.95, 106.43, 56.65, 56.44, 51.28, 50.12, 48.57, 44.41, $41.74,40.48,39.29,38.88,38.36,37.68,37.31,35.92,33.89,33.52,33.39,31.22,30.08,28.95,27.14,26.37$, $24.93,23.15,23.00,21.70,21.15,21.10,20.07,19.61,19.32,19.13,12.68$. HREIMS $m / z 714.5292[\mathrm{M}+\mathrm{H}]^{+}$ (calcd for $\mathrm{C}_{45} \mathrm{H}_{68} \mathrm{~N}_{3} \mathrm{O}_{4}, 714.5210$ ).

Compound (6). Colorless resin; $[\alpha]_{D}^{20}+33\left(c 0.085, \mathrm{CHCl}_{3}\right)$; IR (film) $v_{\max } 2949,2864,1754,1719$, 1678, 1459, 1124, $750 \mathrm{~cm}^{-1}$; ${ }^{1} \mathrm{H}-\mathrm{NMR}\left(400 \mathrm{MHz}, \mathrm{CDCl}_{3}\right) \delta 7.31$ (s, 1H, H-1'), 6.93 (s, 1H, H-14'), 6.80 (s, 1H, H-11" ), 4.82 (s, 1H, H-17), 4.44 (s, 1H, H-17), 4.36-4.25 (m, 2H, H-15), 3.60 (s, 1H, OMe), 2.93-2.75 $\left(\mathrm{m}, 3 \mathrm{H}, \mathrm{H}-15^{\prime \prime}, \mathrm{H}-7^{\prime \prime}\right), 2.63\left(\mathrm{t}, J=7.4 \mathrm{~Hz}, 2 \mathrm{H}, \mathrm{H}-5^{\prime}\right), 2.38(\mathrm{~m}, 1 \mathrm{H}, \mathrm{H}-7), 2.17-2.10(\mathrm{~m}, 2 \mathrm{H}, \mathrm{H}-3$ and $\left.\mathrm{H}-1 \beta^{\prime \prime}\right), 2.14\left(\mathrm{t}, J=7.4 \mathrm{~Hz}, 2 \mathrm{H}, \mathrm{H}-3^{\prime}\right), 1.17(\mathrm{~s}, 3 \mathrm{H}, \mathrm{H}-18), 1.16\left(\mathrm{~s}, 3 \mathrm{H}, \mathrm{H}-20^{\prime \prime}\right), 1.16(\mathrm{~d}, J=6.6 \mathrm{~Hz}, 3 \mathrm{H}$, H-16"), 1.15 (d, J = 6.6 Hz, 3H, H-17"'), 0.94 (d, J = 6.6 Hz, 3H, H-16), 0.93 (s, 3H, H-18' ), 0.90 (s, 3H, H-19"), 0.47 (s, 1H, H-20). ${ }^{13}$ C-NMR (101 MHz, $\left.\mathrm{CDCl}_{3}\right) \delta$ 177.86, 172.29, 148.86, 148.37, 147.08, 146.15, 136.62 , 133.12, 126.91, 120.82, 118.00, 106.39, 56.66, 56.44, 51.24, 50.13, 48.54, 44.40, 41.73, 40.47, 39.28, 
$38.87,38.35,37.67,37.31,35.93,33.70,33.51,33.37,31.19,30.06,28.93,27.18,26.35,25.09,24.89,24.82$, $23.20,23.05,21.68,21.12,20.07,19.60,19.30,19.13,12.67$. HREIMS $m / z 728.5332[\mathrm{M}+\mathrm{H}]^{+}$(calcd for $\left.\mathrm{C}_{46} \mathrm{H}_{70} \mathrm{~N}_{3} \mathrm{O}_{4}, 728.5366\right)$.

Compound (7). Colorless resin; $[\alpha]_{D}^{20}+57\left(c 0.122, \mathrm{CHCl}_{3}\right)$; IR (film) $v_{\max } 2955,2867,1798,1760$, 1722, 1648, 1440, 1123, $756 \mathrm{~cm}^{-1}{ }^{1}{ }^{1} \mathrm{H}-\mathrm{NMR}\left(400 \mathrm{MHz}, \mathrm{CDCl}_{3}\right) \delta 7.49\left(\mathrm{~s}, 1 \mathrm{H}, \mathrm{H}-1^{\prime}\right), 6.68\left(\mathrm{~s}, 1 \mathrm{H}, \mathrm{H}-14^{\prime \prime}\right)$, 4.77 (s, 1H, H-17), 4.41 (s, 1H, H-17), 4.35-4.22 (m, 2H, H-15), $3.56(\mathrm{~s}, 3 \mathrm{H}, \mathrm{OMe}), 3.15(\mathrm{t}, J=6.6 \mathrm{~Hz}, 2 \mathrm{H}$ $\left.\mathrm{H}-4^{\prime}\right), 2.97\left(\mathrm{t}, J=7.5 \mathrm{~Hz}, 2 \mathrm{H}, \mathrm{H}-3^{\prime}\right), 2.95-2.89\left(\mathrm{~m}, 1 \mathrm{H}, \mathrm{H}-15^{\prime \prime}\right), 2.59\left(\mathrm{~m}, 2 \mathrm{H}, \mathrm{H}-7^{\prime \prime}\right), 2.36-2.32(\mathrm{~m}, 1 \mathrm{H}, \mathrm{H}-7)$, 1.15 (d, J = 7.3 Hz, 3H, H-16"), 1.14 (s, 3H, H-18), 1.11 (s, 3H, H-18'), 1.08 (d, J = 6.9 Hz, 3H, H-17"), $1.04\left(\mathrm{~s}, 3 \mathrm{H}, \mathrm{H}-19^{\prime \prime}\right), 0.90$ (d, J = 6.5 Hz, 3H, H-16), 0.44 (s, 3H, H-20). ${ }^{13} \mathrm{C}-\mathrm{NMR}\left(101 \mathrm{MHz}, \mathrm{CDCl}_{3}\right) \delta$ $177.71,177.49,170.20,148.17,144.53,140.83$, 137.25, 130.35, 129.38, 121.53, 119.97, 106.35, 56.54, 56.43, $56.34,51.09,49.57,48.48,44.29,41.58,40.35,39.16,38.77,38.65,38.26,37.10,35.83,33.60,32.85,32.76$, $31.83,31.06,28.83,27.69,26.25,23.92,23.46,22.54,22.17,21.17,20.98,19.97,19.45,18.18,12.56$. HREIMS $m / z 742.4739[\mathrm{M}+\mathrm{H}]^{+}$(calcd for $\left.\mathrm{C}_{45} \mathrm{H}_{64} \mathrm{~N}_{3} \mathrm{O}_{6}, 742.4795\right)$.

Compound (8). Colorless resin; $[\alpha]_{D}^{20}+56\left(c 0.122, \mathrm{CHCl}_{3}\right)$; IR (film) $v_{\max } 2949,2864,1798,1763$, 1720, 1650, 1440, 1123, $756 \mathrm{~cm}^{-1},{ }^{1} \mathrm{H}-\mathrm{NMR}\left(400 \mathrm{MHz}, \mathrm{CDCl}_{3}\right) \delta 7.39$ (s, $\left.1 \mathrm{H}, \mathrm{H}-1^{\prime}\right), 6.69$ (s, $\left.1 \mathrm{H}, \mathrm{H}-14^{\prime \prime}\right)$, 4.78 (s, 1H, H-17), 4.40 (s, 1H, H-17), 4.40-4.25 (m, 2H, H-15), 3.56 (s, 3H, OMe), 2.97 (m, 1H, H-15"), $2.84\left(\mathrm{t}, J=7.3 \mathrm{~Hz}, 2 \mathrm{H}, \mathrm{H}-5^{\prime}\right), 2.62\left(\mathrm{t}, J=7.3 \mathrm{~Hz}, 2 \mathrm{H}, \mathrm{H}-3^{\prime}\right), 2.61-2.58\left(\mathrm{~m}, 2 \mathrm{H}, \mathrm{H}-7^{\prime \prime}\right), 2.39-2.29(\mathrm{~m}, 1 \mathrm{H}$, H-7), $1.18\left(\mathrm{~d}, J=6.9 \mathrm{~Hz}, 3 \mathrm{H} \mathrm{H}-16^{\prime \prime}\right), 1.13(\mathrm{~s}, 3 \mathrm{H}, \mathrm{H}-18), 1.12\left(\mathrm{~d}, J=6.9 \mathrm{~Hz}, 3 \mathrm{H} \mathrm{H}-17^{\prime \prime}\right), 1.11\left(\mathrm{~s}, 3 \mathrm{H}, \mathrm{H}-18^{\prime \prime}\right)$, 1.04 (s, 3H, H-19") 0.91 (d, J = 6.5 Hz, 3H, H-16), 0.44 (s, 1H, H-20). ${ }^{13} \mathrm{C}-\mathrm{NMR}\left(101 \mathrm{MHz}, \mathrm{CDCl}_{3}\right) \delta$ $177.69,177.63$, 170.65, 148.18, 144.60, 140.87, 137.15, 130.36, 129.48, 121.23, 119.91, 106.33, 56.55, 56.42, $56.33,51.09,49.59,48.40,44.28,41.55,40.35,39.16,38.76,38.63,38.25,37.13,35.83,32.88,32.84,32.77$, $31.82,31.05,28.82,27.68,26.25,24.59,24.54,23.93,23.52,22.60,22.17,20.98,19.96,19.48,18.17,12.57$. HREIMS $m / z 756.4894[\mathrm{M}+\mathrm{H}]^{+}$(calcd for $\mathrm{C}_{46} \mathrm{H}_{66} \mathrm{~N}_{3} \mathrm{O}_{6}, 756.4952$ ).

Compound (9). White solid; $[\alpha]_{D}^{20}+30\left(c 0.052, \mathrm{CHCl}_{3}\right)$; IR (film) $v_{\max } 3407,2949,2870,1720,1691$, 1462, 1152, $756 \mathrm{~cm}^{-1},{ }^{1} \mathrm{H}-\mathrm{NMR}\left(400 \mathrm{MHz}, \mathrm{CDCl}_{3}\right) \delta 7.30$ (s, 1H, H-1'), 5.25 (brs, $\left.1 \mathrm{H}, \mathrm{H}-12^{\prime \prime}\right), 4.82(\mathrm{~s}, 1 \mathrm{H}$, $\mathrm{H}-17), 4.47\left(\mathrm{t}, J=7.9 \mathrm{~Hz}, 1 \mathrm{H}, \mathrm{H}-3 \alpha^{\prime \prime}\right), 4.43$ (s, 1H, H-17), 4.38-4.25 (m, 2H, H-15), 3.60 (s, 3H, OMe), 3.03 $\left(\mathrm{t}, J=7.3 \mathrm{~Hz}, 2 \mathrm{H}, \mathrm{H}-4^{\prime}\right), 2.80\left(\mathrm{dd}, J=13.6,3.7 \mathrm{~Hz}, 1 \mathrm{H}, \mathrm{H}-18^{\prime \prime}\right), 2.71\left(\mathrm{t}, J=7.0 \mathrm{~Hz}, 2 \mathrm{H}, \mathrm{H}-3^{\prime}\right), 2.39-2.36$ (m, 1H, H-7), 2.17-2.14 (m, 1H, H-3), 1.17 (s, 3H, H-18), 1.11 (s, 3H), 0.93 (d, J = 6.6 Hz, 3H, H-16), $0.91(\mathrm{~s}, 6 \mathrm{H}), 0.89(\mathrm{~s}, 3 \mathrm{H}), 0.80(\mathrm{~s}, 3 \mathrm{H}), 0.78(\mathrm{~s}, 3 \mathrm{H}), 0.72(\mathrm{~s}, 3 \mathrm{H}), 0.47(\mathrm{~s}, 3 \mathrm{H}, \mathrm{H}-20) .{ }^{13} \mathrm{C}-\mathrm{NMR}(101 \mathrm{MHz}$, $\left.\mathrm{CDCl}_{3}\right) \delta 183.93,177.87,172.72,148.36,146.51,143.76,122.58,121.05,106.42,81.21,56.67,56.45,55.41$, $51.28,48.53,47.65,46.62,45.96,44.41,41.66,41.02,40.48,39.37,39.30,38.89,38.36,38.15,37.82,37.30$, $37.07,35.96,34.23,33.89,33.18,32.63,32.54,31.16,30.78,28.95,28.11,27.77,26.36,26.02,23.69,23.65$, $23.49,22.98,21.29,21.09,20.08,19.60,18.26,17.24,16.80,15.47,12.69$. HREIMS $m / z 898.6106[\mathrm{M}+\mathrm{H}]^{+}$ (calcd for $\mathrm{C}_{56} \mathrm{H}_{88} \mathrm{~N}_{3} \mathrm{O}_{6}, 898.6673$ ).

Compound (10). White solid; $[\alpha]_{D}^{20}+26\left(c 0.064, \mathrm{CHCl}_{3}\right)$; IR (film) $v_{\max } 2938,2875,1724,1458,1157$, $752 \mathrm{~cm}^{-1} ;{ }^{1} \mathrm{H}-\mathrm{NMR}\left(400 \mathrm{MHz}, \mathrm{CDCl}_{3}\right) \delta 7.29\left(\mathrm{~s}, 1 \mathrm{H}, \mathrm{H}-1^{\prime}\right), 5.26$ (brs, $\left.1 \mathrm{H}, \mathrm{H}-12^{\prime \prime}\right), 4.81$ (s, 1H, H-17), 4.47 $\left(\mathrm{t}, J=7.9 \mathrm{~Hz}, 1 \mathrm{H}, \mathrm{H}-3 \alpha^{\prime \prime}\right), 4.42(\mathrm{~s}, 1 \mathrm{H}, \mathrm{H}-17), 4.38-4.24(\mathrm{~m}, 2 \mathrm{H}, \mathrm{H}-15), 3.60$ (s, 3H, OMe), 3.59 (s, 3H, OMe), $3.02\left(\mathrm{t}, J=7.3 \mathrm{~Hz}, 2 \mathrm{H}, \mathrm{H}-4^{\prime}\right), 2.84\left(\mathrm{dd}, J=13.7,3.6 \mathrm{~Hz}, 1 \mathrm{H}, \mathrm{H}-18^{\prime \prime}\right), 2.70\left(\mathrm{t}, J=7.0 \mathrm{~Hz}, 2 \mathrm{H}, \mathrm{H}-3^{\prime}\right)$, 2.39-2.36 (m, 1H, H-7), 2.17-2.13 (m, 1H, H-3), 1.16 (s, 3H, H-18), $1.10(\mathrm{~s}, 3 \mathrm{H}), 0.92(\mathrm{~d}, J=6.6 \mathrm{~Hz}, 3 \mathrm{H}$, $\mathrm{H}-16), 0.90(\mathrm{~s}, 6 \mathrm{H}), 0.88(\mathrm{~s}, 3 \mathrm{H}), 0.80(\mathrm{~s}, 3 \mathrm{H}), 0.77(\mathrm{~s}, 3 \mathrm{H}), 0.70(\mathrm{~s}, 3 \mathrm{H}), 0.46(\mathrm{~s}, 3 \mathrm{H}, \mathrm{H}-20) .{ }^{13} \mathrm{C}-\mathrm{NMR}$ $\left(101 \mathrm{MHz}, \mathrm{CDCl}_{3}\right) \delta 178.37,177.83,172.67,148.35,146.50,143.91,122.34,120.98,106.40,81.20,56.65$, $56.43,55.41,51.62,51.25,48.49,47.64,46.80,45.94,44.39,41.72,41.38,40.46,39.37,39.28,38.87,38.35$, $38.18,37.80,37.28,37.01,35.94,34.24,33.94,33.20,32.68,32.46,31.13,30.78,28.94,28.09,27.77,26.35$, $26.00,23.74,23.64,23.49,23.15,21.31,21.08,20.07,19.59,18.29,16.92,16.80,15.44,12.67$. HREIMS $\mathrm{m} / \mathrm{z}$ $934.6866[\mathrm{M}+\mathrm{Na}]^{+}$(calcd for $\left.\mathrm{C}_{57} \mathrm{H}_{89} \mathrm{~N}_{3} \mathrm{NaO}_{6}, 934.6649\right)$.

Compound (11). White solid; $[\alpha]_{D}^{20}+40\left(c 0.123, \mathrm{CHCl}_{3}\right)$; IR (film) $v_{\max } 3406,2943,2871,1719,1688$, 1458, 1150, $752 \mathrm{~cm}^{-1}$; ${ }^{1} \mathrm{H}-\mathrm{NMR}\left(400 \mathrm{MHz}, \mathrm{CDCl}_{3}\right) \delta 7.27$ (s, 1H, H-1'), 5.24 (brs, 1H, H-12" $), 4.80$ (s, 1H, $\mathrm{H}-17), 4.48\left(\mathrm{t}, J=7.9 \mathrm{~Hz}, 1 \mathrm{H}, \mathrm{H}-3 \alpha^{\prime \prime}\right), 4.41(\mathrm{~s}, 1 \mathrm{H}, \mathrm{H}-17), 4.36-4.24(\mathrm{~m}, 2 \mathrm{H}, \mathrm{H}-15), 3.58$ (s, 3H, OMe), 2.80 $\left(\mathrm{dd}, J=13.7,4.0 \mathrm{~Hz}, 1 \mathrm{H}, \mathrm{H}-18^{\prime \prime}\right), 2.73\left(\mathrm{t}, J=7.5 \mathrm{~Hz}, 2 \mathrm{H}, \mathrm{H}-5^{\prime}\right), 2.39-2.37(\mathrm{~s}, 1 \mathrm{H}, \mathrm{H}-7), 2.34(\mathrm{t}, J=7.3 \mathrm{~Hz}$, 2H, H-3'), 2.16-2.12 (m, 1H, H-3), 1.15 (s, 3H, H-18), 1.10 (s, 3H), 0.92 (d, J = 6.6 Hz, 3H, H-16), 0.90 
(s, 6H), 0.87 (s, 3H), $0.83(\mathrm{~s}, 3 \mathrm{H}), 0.82(\mathrm{~s}, 3 \mathrm{H}), 0.72(\mathrm{~s}, 3 \mathrm{H}), 0.45$ (s, 3H, H-20). ${ }^{13} \mathrm{C}-\mathrm{NMR}(101 \mathrm{MHz}$, $\left.\mathrm{CDCl}_{3}\right) \delta 184.00,177.85,173.24,148.32,147.18,143.74,122.52,120.78,106.38,80.93,56.60,56.39,55.34$, 51.24, 48.50, 47.61, 46.57, 45.92, 44.36, 41.62, 40.96, 40.43, 39.33, 39.24, 38.84, 38.31, 38.10, 37.79, 37.26, $37.04,35.89,34.10,33.86,33.15,32.58,32.50,31.13,30.75,28.91,28.17,27.73,26.32,25.99,25.03,24.92$, $23.66,23.64,23.45,22.93,21.07,20.03,19.57,18.23,17.20,16.83,15.45,12.65$. HREIMS $m / z 934.6572$ $[\mathrm{M}+\mathrm{Na}]^{+}$(calcd for $\mathrm{C}_{57} \mathrm{H}_{89} \mathrm{~N}_{3} \mathrm{NaO}_{6}, 934.6649$ ).

Compound (12). White solid; $[\alpha]_{D}^{20}+19\left(c 0.012, \mathrm{CHCl}_{3}\right)$; IR (film) $v_{\max } 2947,2862,1724,1454,1153$, $752 \mathrm{~cm}^{-1} ;{ }^{1} \mathrm{H}-\mathrm{NMR}\left(400 \mathrm{MHz}, \mathrm{CDCl}_{3}\right) \delta 7.27\left(\mathrm{~s}, 1 \mathrm{H}, \mathrm{H}-1^{\prime}\right), 5.27$ (brs, 1H, H-12') 4.82 (s, 1H, H-17), 4.49 $\left(\mathrm{t}, J=7.9 \mathrm{~Hz}, 1 \mathrm{H}, \mathrm{H}-3 \alpha^{\prime \prime}\right), 4.43$ (s, 1H, H-17), 4.36-4.25 (m, 2H, H-15), 3.61 (s, 3H, OMe), $3.60(\mathrm{~s}, 3 \mathrm{H}$, OMe), $2.85\left(\mathrm{dd}, J=13.6,3.8 \mathrm{~Hz}, 1 \mathrm{H}, \mathrm{H}-18^{\prime \prime}\right), 2.75\left(\mathrm{t}, J=7.5 \mathrm{~Hz}, 2 \mathrm{H}, \mathrm{H}-5^{\prime}\right), 2.40-2.37(\mathrm{~s}, 1 \mathrm{H}, \mathrm{H}-7), 2.36$ $\left(\mathrm{t}, J=7.2 \mathrm{~Hz}, 2 \mathrm{H}, \mathrm{H}-3^{\prime}\right), 2.17-2.14(\mathrm{~m}, 1 \mathrm{H}, \mathrm{H}-3), 1.17(\mathrm{~s}, 3 \mathrm{H}, \mathrm{H}-18), 1.12(\mathrm{~s}, 3 \mathrm{H}), 0.94(\mathrm{~d}, J=6.6 \mathrm{~Hz}, 3 \mathrm{H}$, $\mathrm{H}-16), 0.91(\mathrm{~s}, 6 \mathrm{H}), 0.89(\mathrm{~s}, 3 \mathrm{H}), 0.84(\mathrm{~s}, 6 \mathrm{H}), 0.71(\mathrm{~s}, 3 \mathrm{H}), 0.47$ (s, 3H, H-20). ${ }^{13} \mathrm{C}-\mathrm{NMR}\left(101 \mathrm{MHz}, \mathrm{CDCl}_{3}\right)$ $\delta 178.45,177.91,173.27,148.40,147.27,143.92,122.37,120.76,106.41,80.99,56.66,56.44,55.40,51.67$, $51.29,48.53,47.66,46.83,45.95,44.41,41.74,41.39,40.49,39.39,39.30,38.89,38.36,38.19,37.85,37.32$, $37.04,35.95,34.17,33.97,33.23,32.69,32.49,31.19,30.81,28.96,28.22,27.79,26.37,26.02,25.13,24.98$, 23.76, 23.70, 23.52, 23.17, 21.13, 20.08, 19.62, 18.32, 16.94, 16.90, 15.48, 12.69. HREIMS $m / z$ 926.7061 $[\mathrm{M}+\mathrm{H}]^{+}$(calcd for $\left.\mathrm{C}_{58} \mathrm{H}_{92} \mathrm{~N}_{3} \mathrm{O}_{6}, 926.6986\right)$.

Compound (13). White solid; $[\alpha]_{D}^{20}+24\left(c 0.062, \mathrm{CHCl}_{3}\right)$; IR (film) $v_{\max } 3433,2934,2857,1733,1679$, 1472, 1153, $752 \mathrm{~cm}^{-1} ;{ }^{1} \mathrm{H}-\mathrm{NMR}\left(400 \mathrm{MHz}, \mathrm{CDCl}_{3}\right) \delta 7.30\left(\mathrm{~s}, 1 \mathrm{H}, \mathrm{H}-1^{\prime}\right), 5.49(\mathrm{dd}, J=7.7,3.1 \mathrm{~Hz}, 1 \mathrm{H}$, H-15"), 4.81 (s, 1H, H-17), 4.46-4.42 (m, 1H, H-3"), 4.42 (s, 1H, H-17), 4.34-4.22 (m, 2H, H-15), 3.58 $(\mathrm{s}, 3 \mathrm{H}, \mathrm{OMe}), 3.01\left(\mathrm{t}, J=7.3 \mathrm{~Hz}, 2 \mathrm{H}, \mathrm{H}-4^{\prime}\right), 2.69\left(\mathrm{t}, J=7.0 \mathrm{~Hz}, 2 \mathrm{H}, \mathrm{H}-3^{\prime}\right), 2.39-2.32(\mathrm{~m}, 2 \mathrm{H}, \mathrm{H}-7$ and H-16" , 2.27-2.23 (m, 1H, H-18"), 2.16-2.13 (m, 1H, H-3), 1.16 (s, 3H, H-18), 0.92 (d, J = 6.6 Hz, 3H, H-16), $0.91(\mathrm{~s}, 6 \mathrm{H}), 0.90(\mathrm{~s}, 3 \mathrm{H}), 0.89(\mathrm{~s}, 6 \mathrm{H}), 0.83(\mathrm{~s}, 3 \mathrm{H}), 0.76(\mathrm{~s}, 3 \mathrm{H}), 0.46(\mathrm{~s}, 3 \mathrm{H}, \mathrm{H}-20) .{ }^{13} \mathrm{C}-\mathrm{NMR}(101 \mathrm{MHz}$, $\left.\mathrm{CDCl}_{3}\right) \delta 184.45,177.83,172.65,160.53,148.31,146.45,121.03,116.98,106.40,81.11,56.61,56.40,55.64$, 51.56, 51.25, 49.12, 48.49, 44.36, 41.40, 40.78, 40.44, 39.25, 39.08, 38.85, 38.33, 37.97, 37.77, 37.41, 37.36, $37.26,35.90,35.40,34.18,33.73,33.37,31.94,31.37,31.09,30.76,29.38,28.92,28.74,28.01,26.32,26.27$, $23.54,22.56,21.25,21.04,20.04,19.56,18.77,17.39,16.70,15.70,12.65$. HREIMS $m / z 898.6540[\mathrm{M}+\mathrm{H}]^{+}$ (calcd for $\mathrm{C}_{56} \mathrm{H}_{88} \mathrm{~N}_{3} \mathrm{O}_{6}, 898.6673$ ).

Compound (14). Colorless resin; $[\alpha]_{D}^{20}+22\left(c 0.056, \mathrm{CHCl}_{3}\right)$; IR (film) $v_{\max } 2943,2871,1724,1454$, 1166, $752 \mathrm{~cm}^{-1} ;{ }^{1} \mathrm{H}-\mathrm{NMR}\left(400 \mathrm{MHz}, \mathrm{CDCl}_{3}\right) \delta 7.29\left(\mathrm{~s}, 1 \mathrm{H}, \mathrm{H}-1^{\prime}\right), 5.48\left(\mathrm{dd}, J=7.9,3.3 \mathrm{~Hz}, 1 \mathrm{H}, \mathrm{H}-15^{\prime \prime}\right)$, 4.81 (s, 1H, H-17), 4.46-4.43 (m, 1H, H-3") 4.43 (s, 1H, H-17), 4.31-4.24 (m, 2H, H-15), 3.60 (s, 3H, $\mathrm{OMe}), 3.55(\mathrm{~s}, 3 \mathrm{H}, \mathrm{OMe}), 3.02\left(\mathrm{t}, J=7.3 \mathrm{~Hz}, 2 \mathrm{H}, \mathrm{H}-4^{\prime}\right), 2.70\left(\mathrm{t}, J=7.7 \mathrm{~Hz}, 2 \mathrm{H}, \mathrm{H}-3^{\prime}\right), 2.34-2.34(\mathrm{~m}, 2 \mathrm{H}$, H-7 and H-16" , 2.32-2.29 (m, 1H, H-18' ), 2.17-2.14 (m, 1H, H-3), 1.17 (s, 3H, H-18), 0.93 (d, J = 6.6 Hz, 3H, H-16), $0.92(\mathrm{~s}, 3 \mathrm{H}), 0.91(\mathrm{~s}, 6 \mathrm{H}), 0.90(\mathrm{~s}, 6 \mathrm{H}), 0.82(\mathrm{~s}, 3 \mathrm{H}), 0.76(\mathrm{~s}, 3 \mathrm{H}), 0.47$ (s, 3H, H-20). ${ }^{13} \mathrm{C}-\mathrm{NMR}$ $\left(101 \mathrm{MHz}, \mathrm{CDCl}_{3}\right) \delta 178.57,177.85,172.67,160.61,148.37,146.52,120.99,116.71,106.42,81.26,56.69$, $56.47,55.76,51.80,51.47,51.25,49.15,48.51,44.42,41.97,41.07,40.49,39.31,39.08,38.90,38.38,37.97$, $37.82,37.52,37.50,37.29,35.96,35.60,34.25,33.87,33.52,32.18,31.78,31.15,31.10,29.39,28.95,28.83$, $28.03,26.37,26.36,23.59,22.50,21.32,21.09,20.09,19.60,18.70,17.43,16.69,15.62,12.69$. HREIMS $\mathrm{m} / \mathrm{z}$ $912.6757[\mathrm{M}+\mathrm{H}]^{+}$(calcd for $\left.\mathrm{C}_{57} \mathrm{H}_{90} \mathrm{~N}_{3} \mathrm{O}_{6}, 912.6830\right)$.

Compound (15). White solid; $[\alpha]_{D}^{20}+26\left(c 0.044, \mathrm{CHCl}_{3}\right)$; IR (film) $\nu_{\max } 3433,2938,2862,1728,1679$, 1459, 1153, $757 \mathrm{~cm}^{-1} ;{ }^{1} \mathrm{H}-\mathrm{NMR}\left(400 \mathrm{MHz}, \mathrm{CDCl}_{3}\right) \delta 7.24\left(\mathrm{~s}, 1 \mathrm{H}, \mathrm{H}-1^{\prime}\right), 5.50(\mathrm{dd}, J=7.7,3.1 \mathrm{~Hz}, 1 \mathrm{H}$, H-15" ), 4.80 (s, 1H, H-17), 4.44-4.41 (m, 1H, H-3") 4.41 (s, 1H, H-17), 4.33-4.23 (m, 2H, H-15), 3.58 (s, 3H, $\mathrm{OMe}), 2.70\left(\mathrm{t}, J=7.5 \mathrm{~Hz}, 2 \mathrm{H}, \mathrm{H}-5^{\prime}\right), 2.40-2.22\left(\mathrm{~m}, 5 \mathrm{H}, \mathrm{H}-7, \mathrm{H}-16^{\prime \prime}, \mathrm{H}-5^{\prime}\right.$ and $\left.\mathrm{H}-18^{\prime \prime}\right), 2.16-2.12(\mathrm{~m}, 1 \mathrm{H}$, H-3), 1.15 (s, 3H, H-18), $0.92(\mathrm{~s}, 6 \mathrm{H}), 0.91$ (d, J = 6.6 Hz, 3H, H-16), $0.90(\mathrm{~s}, 3 \mathrm{H}), 0.89$ (s, 6H), $0.84(\mathrm{~s}, 3 \mathrm{H})$, $0.81(\mathrm{~s}, 3 \mathrm{H}), 0.46$ (s, 3H, H-20). ${ }^{13} \mathrm{C}-\mathrm{NMR}\left(101 \mathrm{MHz}, \mathrm{CDCl}_{3}\right) \delta 184.28,177.87,173.47,160.63,148.39$, 147.80, 120.54, 116.97, 106.40, 80.82, 56.66, 56.46, 55.70, 51.57, 51.26, 49.18, 48.51, 44.41, 41.49, 40.87, $40.48,39.30,39.14,38.89,38.37,38.03,37.81,37.48,37.41,37.32,35.94,35.46,34.50,33.79,33.42,31.98$, $31.44,31.20,30.82,29.41,28.97,28.95,28.77,28.12,26.37,26.29,25.45,24.71,22.58,21.13,20.09,19.61$, $18.82,17.43,16.77,15.74,12.69$. HREIMS $m / z 934.6755[\mathrm{M}+\mathrm{Na}]^{+}\left(\right.$calcd for $\left.\mathrm{C}_{57} \mathrm{H}_{89} \mathrm{~N}_{3} \mathrm{NaO}_{6}, 934.6649\right)$. 
Compound (16). Colorless resin; $[\alpha]_{D}^{20}+40\left(c 0.030, \mathrm{CHCl}_{3}\right)$; IR (film) $v_{\max } 2938,2862,1724,1454$, 1157, $752 \mathrm{~cm}^{-1}$; ${ }^{1} \mathrm{H}-\mathrm{NMR}\left(400 \mathrm{MHz}, \mathrm{CDCl}_{3}\right) \delta 7.24\left(\mathrm{~s}, 1 \mathrm{H}, \mathrm{H}-1^{\prime}\right), 5.49\left(\mathrm{dd}, J=7.9,3.3 \mathrm{~Hz}, 1 \mathrm{H}, \mathrm{H}-15^{\prime \prime}\right)$, 4.82 (s, 1H, H-17), 4.47-4.43 (m, 1H, H-3") 4.44 (s, 1H, H-17), 4.33-4.26 (m, 2H, H-15), 3.60 (s, 3H, $\mathrm{OMe}), 3.56(\mathrm{~s}, 3 \mathrm{H}, \mathrm{OMe}), 2.72\left(\mathrm{t}, J=6.8 \mathrm{~Hz}, 1 \mathrm{H}, \mathrm{H}-5^{\prime}\right), 2.41-2.31\left(\mathrm{~m}, 5 \mathrm{H}, \mathrm{H}-7, \mathrm{H}-16^{\prime \prime}, \mathrm{H}-5^{\prime}\right.$ and $\left.\mathrm{H}-18^{\prime \prime}\right)$, 2.18-2.15 (m, 1H, H-3), 1.17 (s, 3H, H-18), 0.94 (d, J = 6.6 Hz, 3H, H-16), 0.93 (s, 3H), 0.92 (s, 9H), 0.91 $(\mathrm{s}, 3 \mathrm{H}), 0.84(\mathrm{~s}, 3 \mathrm{H}), 0.82(\mathrm{~s}, 3 \mathrm{H}), 0.48(\mathrm{~s}, 3 \mathrm{H}, \mathrm{H}-20) .{ }^{13} \mathrm{C}-\mathrm{NMR}\left(101 \mathrm{MHz}, \mathrm{CDCl}_{3}\right) \delta 178.62,177.90,173.48$, $160.65,148.43,147.85,124.43,120.52,116.72,106.41,80.93,56.70,56.49,55.77,51.83,51.49,51.28,49.16$, 48.52 , 44.44, 41.99, 41.10, 40.51, 39.33, 39.11, 38.92, 38.40, 38.01, 37.84, 37.52, 37.35, 36.77, 35.97, 35.62, $34.58,33.89,33.55,32.20,31.81,31.22,31.13,29.41,29.01,28.97,28.85,28.14,26.40,26.38,25.49,24.75$, $22.52,21.15,20.11,19.64,18.73,17.45,16.77,15.65,12.71$. HREIMS $m / z 926.7031[\mathrm{M}+\mathrm{H}]^{+}$(calcd for $\left.\mathrm{C}_{58} \mathrm{H}_{92} \mathrm{~N}_{3} \mathrm{O}_{6}, 926.6986\right)$.

\subsection{Treatment Solutions}

The synthetic compounds and acyclovir (ACV, Sigma) were dissolved in dimethylsulfoxide (DMSO) and diluted with Eagle's minimal essential medium supplemented with 1.5\% inactivated fetal bovine serum (FBS) and $50 \mu \mathrm{g} / \mathrm{mL}$ gentamicin (MEM 1.5\%). The maximum concentration of DMSO used $(1 \%)$ exhibited no toxicity under in vitro conditions.

\subsection{Cells and Viruses}

Vero and Hep-2 cells were grown in MEM supplemented with $10 \%$ inactivated FBS and $50 \mu \mathrm{g} / \mathrm{mL}$ gentamicin and maintained in MEM 1.5\%. Raw 264.7 cells were grown in Dulbecco's Modified Eagle's Medium (DMEM) supplemented with 10\% FBS and $50 \mu \mathrm{g} / \mathrm{mL}$ gentamicin and maintained in DMEM $1.5 \%$. C6 cells were grown in DMEM-F12 supplemented with $2.5 \% \mathrm{FBS} 50 \mu \mathrm{g} / \mathrm{mL}$ gentamicin and $15 \%$ horse serum (HS) and maintained in DMEM-F12 supplemented with 1.5\% FBS. HSV-1 KOS, thymidine kinase-deficient (TK-) B2006 and Field strains of HSV-1 were used and propagated at low multiplicity of infection (m.o.i.) on Vero cells.

\subsection{Cytotoxicity Assay}

Cell viability was determined using the tetrazolium salt MTT (3-(4,5-dimethylthiazol-2-yl) -2,5-diphenyltetrazolium bromide, Sigma) according to the manufacturer's instructions. Hep-2, C6, Raw 264.7 and Vero cells were seeded at a concentration of $1 \times 10^{4}$ cells/well in 96-well plates and grown at $37^{\circ} \mathrm{C}$ for $24 \mathrm{~h}$. The culture medium was replaced by medium containing the compounds in triplicate, and cells were incubated for $48 \mathrm{~h}$. The absorbance was measured on a MPR-A 4i microplate reader (Eurogenetics, Tipperary, Ireland) using a test wavelength of $570 \mathrm{~nm}$ and a reference wavelength of $630 \mathrm{~nm}$. Results were expressed as a percentage of absorbance of treated cell cultures with respect to untreated ones. The cytotoxic concentration $50\left(C_{50}\right)$ was calculated as the concentration of compounds required to reduce cell viability by $50 \%$ relative to untreated cells.

\subsection{Cytopathic Effect Assay}

Cells grown in 96-well plates were infected or not with HSV-1 (KOS, Field and B2006 strains) at a m.o.i. of $0.1 \mathrm{PFU} /$ cell. After 1-h adsorption at $37^{\circ} \mathrm{C}$, the inoculum was removed and medium containing or not the compounds was added, in triplicate. The plates were incubated at $37^{\circ} \mathrm{C}$ until $48 \mathrm{~h}$ post-infection (p.i.), when 100\% of cells were death in virus control, due to the cytophatic effect. Then, cells were fixed with $10 \%$ formaldehyde for $15 \mathrm{~min}$ at room temperature, washed once with distilled water and stained with $0.05 \%$ crystal violet in $10 \%$ ethanol over $30 \mathrm{~min}$. Afterward, cells were washed once and eluted with a solution of $50 \%$ ethanol and $0.1 \%$ acetic acid in water. The absorbance of each well was measured on an Eurogenetics MPR-A 4i microplate reader using a test wave-length of $590 \mathrm{~nm}$. Results were analyzed as the percentage of absorbance of treated and infected cells compared with control (untreated/uninfected) cells. We considered the untreated/uninfected control cells as 
$100 \%$ of cell survival. The compound concentration that inhibit $50 \%$ viral cytopathic effect $\left(\mathrm{EC}_{50}\right)$ was calculated.

\subsection{Statistical Analysis}

The data were expressed as the mean \pm standard deviation (SD) of three independent experiments.

\section{Conclusions}

To expand the structural diversity of naturally occurring terpenes in the search for new compounds of biological interest, sixteen hybrid compounds were synthetized. Using click reactions the diterpene imbricatolic acid (IA) containing an azide group and different terpenes (cyperenoic acid, dehydroabietinol, carnosic acid $\gamma$-lactone, ferruginol, oleanolic acid and aleuritolic acid) containing alkyne groups were fused. The cytotoxic activity of the hybrids was assessed against Vero and selected tumour cell lines. The compounds 1, 2, 3 and $\mathbf{7}$ were the most cytotoxic against the cell lines tested. The antiviral activity of the compounds was evaluated against the KOS, Field and B2006 strain of HSV-1. For the pairs of hybrid compounds 3-8 (except for compound 7), a moderate activity was observed against the three HSV-1 strains with interesting selectivity index for some compounds.

Little information exists in bibliography on the possible activity of fused terpenes so this technique can be applied to a wide range of molecules (terpenes or not) to obtain new hybrid molecules with enhanced pharmacological action.

Author Contributions: M.W.P. was responsible for the synthesis and wrote the manuscript, E.P and L.E.A. carried out the biological assays. G.S.-H. contributed with valuable discussion and revision of the manuscript.

Acknowledgments: M.W.P. thanks to FONDECYT Chile (grant 11100046 and 1161092). E.P and L.E.A. thanks to Universidad de Buenos Aires (UBACYT 20020130100584BA). L.E.A. is a research member of the Consejo Nacional de Investigaciones Científicas y Técnicas (CONICET).

Conflicts of Interest: The authors report no conflict of interest.

\section{References}

1. Arthanari, S.; Vanitha, J.; Krishnaswami, V. In vitro antiviral and cytotoxic screening of methanolic extract of Cassia auriculata flowers in HeLa, Vero, CRFK and HEL cell lines. Drug Invent. Today 2013, 5, 28-31. [CrossRef]

2. Looker, K.J.; Magaret, A.S.; May, M.T.; Turner, K.M.E.; Vickerman, P.; Gottlieb, S.L.; Newman, L.M. Global and regional estimates of prevalent and incident Herpes Simplex Virus type 1 infections in 2012. PLoS ONE 2015, 10, e0140765. [CrossRef] [PubMed]

3. Medini, F.; Legault, J.; Pichette, A.; Abdelly, C.; Ksouri, R. Antiviral efficacy of Limonium densiflorum against HSV-1 and influenza viruses. S. Afr. J. Bot. 2014, 92, 65-72. [CrossRef]

4. Agostini, S.; Mancuso, R.; Baglio, F.; Cabinio, M.; Hernis, A.; Saul, A.; Calabrese, E.; Nemni, R.; Clerici, M. Brain, Behavior, and Immunity High avidity HSV-1 antibodies correlate with absence of amnestic mild cognitive impairment conversion to Alzheimer's disease. Brain Behav. Immun. 2016, 58, 254-260. [CrossRef] [PubMed]

5. Stránská, R.; Schuurman, R.; Nienhuis, E.; Goedegebuure, I.W.; Polman, M.; Weel, J.F.; Dillen, P.M.W.; Berkhout, R.J.M.; Van Loon, A.M. Survey of acyclovir-resistant herpes simplex virus in The Netherlands: Prevalence and characterization. J. Clin. Virol. 2005, 32, 7-18. [CrossRef] [PubMed]

6. Wang, Y.-D.; Zhang, G.-J.; Qu, J.; Li, Y.-H.; Jiang, J.-D.; Liu, Y.-B.; Ma, S.-G.; Li, Y.; Lv, H.-N.; Yu, S.-S. Diterpenoids and sesquiterpenoids from the roots of Illicium majus. J. Nat. Prod. 2013, 76, 1976-1983. [CrossRef] [PubMed]

7. González, M.A.; Zaragozá, R.J. Semisynthesis of the antiviral abietane diterpenoid Jiadifenoic acid C from Callitrisic acid (4-Epidehydroabietic acid) isolated from sandarac resin. J. Nat. Prod. 2014, 77, 2114-2117. [CrossRef] [PubMed]

8. Arnó, M.; Betancur-Galvis, L.; Bueno-Sanchez, J.G.; González, M.A.; Zaragozá, R.J. Synthesis and antiviral activity of scopadulcic acids analogues. Tetrahedron 2003, 59, 6455-6464. [CrossRef] 
9. Oliveira, C.S.; Salvador, M.J.; de Carvalho, J.E.; Santos, É.P.; Barison, A.; Stefanello, M.É.A. Cytotoxic abietane-derivative diterpenoids of Salvia lachnostachys. Phytochem. Lett. 2016, 17, 140-143. [CrossRef]

10. Vila-Luna, S.E.; Moo-Puc, R.E.; Torres-Tapia, L.W.; Peraza-Sánchez, S.R. New metabolites with cytotoxic and antiproliferative activities isolated from Bonellia macrocarpa. Phytochem. Lett. 2017, 19, 121-125. [CrossRef]

11. Pertino, M.; Schmeda-Hirschmann, G.; Rodríguez, J.A.; Theoduloz, C. Gastroprotective effect and cytotoxicity of terpenes from the Paraguayan crude drug "yagua rova" (Jatropha isabelli). J. Ethnopharmacol. 2007, 111, 553-559. [CrossRef] [PubMed]

12. Pertino, M.; Rodríguez, J.A.; Theoduloz, C.; Razmilic, I.; Schmeda-Hirschmann, G. Gastroprotective activity and cytotoxic effect of cyperenoic acid derivatives. J. Pharm. Pharmacol. 2006, 58, 1507-1513. [CrossRef] [PubMed]

13. Pertino, M.; Theoduloz, C.; Butassi, E.; Zacchino, S.; Schmeda-Hirschmann, G. Synthesis, antiproliferative and antifungal activities of 1,2,3-triazole-substituted carnosic acid and carnosol derivatives. Molecules 2015, 20, 8666-8686. [CrossRef] [PubMed]

14. Pertino, M.; Schmeda-Hirschmann, G.; Rodríguez, J.A.; Theoduloz, C. Gastroprotective effect and cytotoxicity of semisynthetic jatropholone derivatives. Planta Med. 2007, 73, 1095-1100. [CrossRef] [PubMed]

15. Theoduloz, C.; Rodríguez, J.A.; Pertino, M.; Schmeda-Hirschmann, G. Antiproliferative activity of the diterpenes jatrophone and jatropholone and their derivatives. Planta Med. 2009, 75, 1520-1522. [CrossRef] [PubMed]

16. Pertino, M.W.; Theoduloz, C.; Bastías, M.; Schmeda-Hirschmann, G. Dimeric labdane diterpenes: Synthesis and antiproliferative effects. Molecules 2013, 18, 5936-5953. [CrossRef] [PubMed]

17. Pertino, M.W.; Theoduloz, C.; Palenzuela, J.A.; Afonso, M.; Yesilada, E.; Monsalve, F.; González, P.; Droguett, D.; Schmeda-hirschmann, G. Synthesis and pharmacological activity of diterpenylnaphthoquinone derivatives. Molecules 2011, 16, 8614-8628. [CrossRef] [PubMed]

18. Viegas-Junior, C.; Danuello, A.; Bolzani, S.; Barreiro, E.J.; Alberto, C.; Fraga, M. Molecular hybridization: A useful tool in the design of new drug prototypes. Curr. Med. Chem. 2007, 14, 1829-1852. [CrossRef] [PubMed]

19. Shaveta, M.S.; Singh, P. Hybrid molecules: The privileged scaffolds for various pharmaceuticals. Eur. J. Med. Chem. 2016, 124, 500-536. [CrossRef] [PubMed]

20. Belluti, F.; Fontana, G.; Bo, L.D.; Carenini, N.; Giommarelli, C.; Zunino, F. Design, synthesis and anticancer activities of stilbene-coumarin hybrid compounds: Identification of novel proapoptotic agents. Bioorganic Med. Chem. 2010, 18, 3543-3550. [CrossRef] [PubMed]

21. Sashidhara, K.V.; Kumar, M.; Modukuri, R.K.; Sonkar, R.; Bhatia, G.; Khanna, A.K.; Rai, S.; Shukla, R. Synthesis and anti-inflammatory activity of novel biscoumarin-chalcone hybrids. Bioorg. Med. Chem. Lett. 2011, 21, 4480-4484. [CrossRef] [PubMed]

22. Sum, T.H.; Sum, T.J.; Galloway, W.R.J.D.; Collins, S.; Twigg, D.G.; Hollfelder, F.; Spring, D.R. Combinatorial synthesis of structurally diverse triazole-bridged flavonoid dimers and trimers. Molecules 2016, 21, 1230. [CrossRef] [PubMed]

23. Tron, G.C.; Pirali, T.; Billington, R.A.; Canonico, P.L.; Sorba, G.; Genazzani, A.A. Click chemistry reactions in medicinal chemistry: Applications of the 1,3-dipolar cycloaddition between azides and alkynes. Med. Res. Rev. 2008, 28, 278-308. [CrossRef] [PubMed]

24. Kategaonkar, A.H.; Shinde, P.V.; Kategaonkar, A.H.; Pasale, S.K.; Shingate, B.B.; Shingare, M.S. Synthesis and biological evaluation of new 2-chloro-3-((4-phenyl-1H-1,2,3-triazol-1-yl)methyl)quinoline derivatives via click chemistry approach. Eur. J. Med. Chem. 2010, 45, 3142-3146. [CrossRef] [PubMed]

25. Chandrashekhar, M.; Nayak, V.L.; Ramakrishna, S.; Mallavadhani, U.V. Novel triazole hybrids of myrrhanone C, a natural polypodane triterpene: Synthesis, cytotoxic activity and cell based studies. Eur. J. Med. Chem. 2016, 114, 293-307. [CrossRef] [PubMed]

26. Anand, A.; Naik, R.J.; Revankar, H.M.; Kulkarni, M.V.; Dixit, S.R.; Joshi, S.D. A click chemistry approach for the synthesis of mono and bis aryloxy linked coumarinyl triazoles as anti-tubercular agents. Eur. J. Med. Chem. 2015, 105, 194-207. [CrossRef] [PubMed]

27. Pete, U.D.; Zade, C.M.; Bhosale, J.D.; Tupe, S.G.; Chaudhary, P.M.; Dikundwar, A.G.; Bendre, R.S. Hybrid molecules of carvacrol and benzoyl urea/thiourea with potential applications in agriculture and medicine. Bioorganic Med. Chem. Lett. 2012, 22, 5550-5554. [CrossRef] [PubMed] 
28. Schmeda-Hirschmann, G.; Pertino, M.W.; Rodriguez, J.A.; Monsalve, F.; Droguett, D.; Theoduloz, C. Synthesis, gastroprotective effect and cytotoxicity of new amino acid diterpene monoamides and diamides. Molecules 2010, 15, 7378-7394. [CrossRef] [PubMed]

29. Marenin, K.S.; Gatilov, Y.V.; Agafontsev, A.M.; Tkachev, A.V. Stereoselectivity of formation of monoterpene-Amino acids hybrid molecules in the reaction of monoterpene nitroso chlorides with $\alpha$-amino acid derivatives. Steroids 2017, 117, 112-119. [CrossRef] [PubMed]

30. Theoduloz, C.; Bravo, I.; Pertino, M.; Valenzuela, D.; Schmeda-Hirschmann, G. Potential gastroprotective effect of novel cyperenoic acid/quinone derivatives in human cell cultures. Planta Med 2012, 78, 1-6. [CrossRef] [PubMed]

31. Theoduloz, C.; Delporte, C.; Valenzuela-Barra, G.; Silva, X.; Cádiz, S.; Bustamante, F.; Pertino, M.W.; Schmeda-Hirschmann, G. Topical anti-inflammatory activity of new hybrid molecules of terpenes and synthetic drugs. Molecules 2015, 20, 11219-11235. [CrossRef] [PubMed]

32. Pawełczyk, A.; Olender, D.; Sowa-Kasprzak, K.; Zaprutko, L. Hybrid compounds strategy in the synthesis of oleanolic acid skeleton-NSAID derivatives. Molecules 2016, 21, 420. [CrossRef] [PubMed]

33. Pertino, M.W.; Verdugo, V.; Theoduloz, C.; Schmeda-hirschmann, G. Synthesis and antiproliferative activity of some novel triazole derivatives from dehydroabietic acid. Molecules 2014, 19, 2523-2535. [CrossRef] [PubMed]

34. Pertino, M.W.; Lopez, C.; Theoduloz, C.; Schmeda-hirschmann, G. 1,2,3-Triazole-substituted oleanolic acid derivatives: Synthesis and antiproliferative activity. Molecules 2013, 18, 7661-7674. [CrossRef] [PubMed]

35. Pertino, M.W.; Theoduloz, C.; Rodríguez, J.A.; Yáñez, T.; Lazo, V.; Schmeda-Hirschmann, G. Gastroprotective effect of carnosic acid gamma-lactone derivatives. J. Nat. Prod. 2010, 73, 639-643. [CrossRef] [PubMed]

36. Areche, C.; Rodríguez, J.A.; Razmilic, I.; Yáñez, T.; Theoduloz, C.; Schmeda-Hirschmann, G. Gastroprotective and cytotoxic effect of semisynthetic ferruginol derivatives. Pharm. Pharmacol. 2006, 59, 289-300. [CrossRef] [PubMed]

37. Sánchez, M.; Theoduloz, C.; Schmeda-Hirschmann, G.; Razmilic, I.; Yáñez, T.; Rodríguez, J.A. Gastroprotective and ulcer-healing activity of oleanolic acid derivatives: In vitro-in vivo relationships. Life Sci. 2006, 79, 1349-1356. [CrossRef] [PubMed]

Sample Availability: Samples of the compounds 1-16 are available from the authors.

(C) 2018 by the authors. Licensee MDPI, Basel, Switzerland. This article is an open access article distributed under the terms and conditions of the Creative Commons Attribution (CC BY) license (http://creativecommons.org/licenses/by/4.0/). 